\title{
BETTEOUI BENYOUNES
}

\section{ABDELILAH GMIRA \\ On the radial solutions of a degenerate quasilinear elliptic equation in $\mathbf{R}^{N}$}

Annales de la faculté des sciences de Toulouse $6^{e}$ série, tome $8, \mathrm{n}^{\circ} 3$ (1999), p. 411-438

<http://www.numdam.org/item?id=AFST_1999_6_8_3_411_0>

(C) Université Paul Sabatier, 1999, tous droits réservés.

L'accès aux archives de la revue «Annales de la faculté des sciences de Toulouse » (http://picard.ups-tlse.fr/ annales/) implique l'accord avec les conditions générales d'utilisation (http://www.numdam.org/conditions). Toute utilisation commerciale ou impression systématique est constitutive d'une infraction pénale. Toute copie ou impression de ce fichier doit contenir la présente mention de copyright.

\section{NumDam}

Article numérisé dans le cadre du programme Numérisation de documents anciens mathématiques http://www.numdam.org/ 


\title{
On the radial solutions of a degenerate quasilinear elliptic equation in $\mathbb{R}^{N(*)}$
}

\author{
Betteoui Benyounes \& AbDelilah Gmira (1)
}

RÉSUMÉ. - On étudie l'existence, l'unicité ainsi que les propriétés qualitatives des solutions radiales de l'équation $\triangle_{p} u+\frac{q+1-p}{p(q-1)} x \nabla u+\frac{u}{q-1}+$ $|u|^{q-1} u=0$ dans $\mathbb{R}^{N}$. Si $q>p-1>1$, alors pour tout $\gamma \in \mathbb{R}^{*}$, il existe une unique radiale solution $u(r, \gamma)$ telle que $u(0, \gamma)=\gamma$. On montre aussi que si $2<p<N, q \geqslant \frac{N p}{N-p}-1$ et $\gamma \in \mathbb{R}_{+}^{*}$ ou bien si $q>\frac{p}{N}+p-1$ et $\gamma$ assez petit, alors $u(r, \gamma)>0$ et $\lim _{r \rightarrow+\infty} r^{\frac{p}{q+1-p}} u(r, \gamma)=c>0$. D'autre part si $p-1<q \leqslant \frac{p}{N}+p-1$ il n'y a pas de solutions strictement positives. De plus si $2<p<N$, et $p-1<q<\frac{N p}{N-p}-1$ alors il existe $\gamma^{*}>0$ tel que pour tout $\gamma \geqslant \gamma^{*}, u(r, \gamma)$ change de signe.

AbSTraCt. - Existence, uniqueness and qualitative behaviour of radial solutions of $\triangle_{p} u+\frac{q+1-p}{p(q-1)} x \nabla u+\frac{u}{q-1}+|u|^{q-1} u=0$ for $x \in \mathbb{R}^{N}$ are presented. If $q>p-1>1$, for any $\gamma \in \mathbb{R}^{*}$ there exists a unique radial solution $u(r, \gamma)$ such that $u(0, \gamma)=\gamma$. Also it is proved that if $2<p<N, q \geqslant \frac{N p}{N-p}-1$ and $\gamma \in \mathbb{R}^{*}$ or if $q>\frac{p}{N}+p-1$ and $\gamma$ small, then $u(r, \gamma)>0$ and $\lim _{r \rightarrow+\infty} r^{\frac{p}{q+1-p}} u(r, \gamma)=c>0$. On the other hand if $p-1<q \leqslant \frac{p}{N}+p-1$ there are no positive solutions. Moreover if $2<p<N$ and $p-1<q<\frac{N p}{N-p}-1$ then there exists $\gamma^{*}>0$ such that for any $\gamma>\gamma^{*}$, the solution $u(r, \gamma)$ can not be positive.

(*) Reçu le 7 septembre 1998, accepté le 1 avril 1999

(1) Départ. de Mathématiques, Faculté des Sciences, B.P. 2121, Tetouan (Maroc). E.mail:gmiraabdelilah@hotmail.com 


\section{Introduction}

This paper is devoted to the study of the quasilinear elliptic equation

$$
\triangle_{p} u+\frac{q+1-p}{p(q-1)} x \nabla u+\frac{1}{q-1} u+|u|^{q-1} u=0, \quad x \in \mathbb{R}^{N} .
$$

where, as usual $\triangle_{p} u=\operatorname{div}\left(|\nabla u|^{p-2} \nabla u\right)$ is the $p$-laplacian and $\nabla u$ is the gradient of $u$. We are concerned with existence, uniqueness and qualitative behavior of radially-symmetric solutions whenever $1<p-1<q$.

The above equation arises naturally in the study of self-similar solutions of the parabolic equation

$$
w_{t}=\triangle_{p} w+|w|^{q-1} w \quad \text { in } \mathbb{R}^{N} \times(0,+\infty)
$$

i.e. solutions $w$ of $(0.2)$ which are of the form

$$
w(x, t)=t^{-\frac{1}{q-1}} u\left(t^{-\frac{q+1-p}{p(q-1)}} x\right)
$$

where $u$ satisfies the equation (0.1). If we make $p=2$ in $(0.2)$ we get the semilinear equation which has been thoroughly studied by Haraux, Weissler, Terman, Peletier ([4], [7], [10]) and others. They noticed the existence of two critical values for $q$ in $(1,+\infty)$ which are $\frac{N+2}{N}$ and $\frac{N+2}{N-2}$ if $N \geqslant 3$ ( 2 if $N=2$ and 3 if $N=1$ ) and they give the precise asymptotic behavior for solutions. Now if we drop the gradient term in (0.1) we obtain a particular case of a large class of equations which have been studied by J. Serrin and P. Pucci [9] . Our purpose here is to extend some results of [4] to the case $p>2$.

It is worth mentioning that if we change the sign in front of the absorption term in (0.2) we get the following equation

$$
w_{t}=\triangle_{p} w-w^{q} \quad \text { in } \mathbb{R}^{N} \times(0,+\infty)
$$

which have been intensively studied. A special attention has been devoted to the study of singular solutions i.e. non negative functions $w$ satisfying $(0.4)$ in $\mathbb{R}^{N} \times(0,+\infty)$ and such that $w(x, 0)=0$ if $x \in \mathbb{R}^{N} \backslash\{0\}$. In fact it was proved by the second author, in [2], that $(0.4)$ has a non trivial singular solution if and only if $0<q<p-1+\frac{P}{N}$. The uniqueness of the fundamental solution i.e. $u(x, 0)=c \delta(x)$ for some positive constant $c$, is proved by Kamin and Vàzquez in [5]. On the other hand, whenever $p-1<q<p-1+\frac{P}{N}$, Peletier and Wang [8] proved the existence of a singular solution satisfying 
On the radial solutions of a degenerate quasilinear elliptic equation in $\mathbb{R}^{N}$

$\lim _{t \rightarrow \infty} \int_{|x|<r} u(x, t) d x=+\infty$ for any $r>0$; which called "very singular solution". Finally Diaz and Saa in [1] studied the uniqueness of a radial solution of following problem

$$
\left\{\begin{array}{l}
\triangle_{p}\left(u^{m}\right)+\frac{q-m(p-1)}{p(q-1)} x \nabla u+\frac{1}{q-1} u-|u|^{q-1} u=0, \quad x \in \mathbb{R}^{N} \\
\lim _{|x| \rightarrow \infty} u(x)=0
\end{array}\right.
$$

where $G(u)=\frac{1}{q-1} u-|u|^{q-1} u$ and $N \geqslant 1, m>0, q>0, p>1$ such that $m(p-1)>1$ and $(p-1) m<q<(p-1) m+\frac{p}{N}$. More precisely they prove that problem (0.5) has at most one solution and this solution has compact support. As a consequence they deduce the uniqueness of the very singular solution of (0.4). From the point of view of the techniques involved in [8], the concrete expression of function $G$ is fundamental. so, we shall not use the shooting method in the phase plane but a different technique.

Since our attention is focused on radial solutions $u(r)$, where $r=|x|$, then the problem (0.1) can be written in the following initial value problem

$$
\left\{\begin{array}{lr}
\left(\left|u^{\prime}\right|^{p-2} u^{\prime}\right)^{\prime}+\frac{N-1}{r}\left|u^{\prime}\right|^{p-2} u^{\prime}+\frac{q+1-p}{p(q-1)} r u^{\prime}+f(u)=0, & r>0 . \\
u(0)=\gamma, u^{\prime}(0)=0, & (0.6)
\end{array}\right.
$$

where $\gamma$ is a constant and

$$
f(u)=\frac{u}{q-1}+|u|^{q-1} u .
$$

By a solution of $(P)$ we mean a function $u \in \mathcal{C}^{1}([0,+\infty))$ such that $\left|u^{\prime}\right|^{p-2} u^{\prime} \in \mathcal{C}^{1}([0,+\infty))$ which satisfies $(0.6)$ and $(0.7)$ for a fixed real $\gamma$.

The following results are obtained.

THEOREM 1. - Let $q \geqslant p-1>1$. Then for any $\gamma \in \mathbb{R}^{*}$, problem $(P)$ has a unique solution $u(r, \gamma)$.

It is important to remark that $u(r,-\gamma)=-u(r, \gamma)$. Therefore, from now on we shall state the results only for $\gamma$ positive.

THEOREM 2. - (i) Assume $q>\frac{p}{N}+p-1$. Then for any

$$
\gamma \in\left(0,\left(\frac{N(q+1-p)}{p(q-1)}-\frac{1}{q-1}\right)^{\frac{1}{q-1}}\right),
$$


the solution $u$ of $(P)$ is strictly positive. Moreover, there is a constant $c$ such that

$$
\lim _{r \rightarrow+\infty} r^{\frac{p}{q+1-p}} u(r, \gamma)=c>0 .
$$

(ii) Assume $2<p<N$ and $q>\frac{N p}{N-1}-1$. Then the conclusion (i) holds for any $\gamma \in \mathbb{R}^{+}$.

TheOREM 3. - (i) Assume $p-1<q \leqslant \frac{p}{N}+p-1$. Then there are no positive solutions of $(P)$.

(ii) Assume $2<p<N$ and $p-1<q<\frac{N p}{N-p}-1$. Then there exists $\gamma^{*}>0$ such that for any $\gamma>\gamma^{*}$, the solution $u$ of $(P)$ can not be positive.

The organization of this paper is as follows.

Section 1 is devoted to the proof of theorem1 which is based on the Banach fixed point theorem.

Section 2 collects some properties of solutions of problem $(P)$. In particular, using an energy function, we prove that any solution $u$ of $(0.6)$ and its derivative $u^{\prime}$ tend to zero as $r$ tends to infinity.

The existence and the asymptotic behavior of strictly positive solutions are studied in section 3 .

Finally, section 4 is devoted to the proof of theorem 3. In part (i) of this theorem we us an estimate of $u^{\prime}$ and part (ii) is proved by using a scaling argument. We also present some important information for non positive solutions. The study of positive rapidly decreasing solutions of $(P)$ remains an open problem.

\section{Existence and uniqueness of solutions}

In this section we investigate the existence and uniqueness of a solution $u(., \gamma)$ of the initial value problem $(P)$ for a fixed $\gamma \in \mathbb{R}^{*}$. Recall that this solution, which we continue denoting by $u$, is a $\mathcal{C}^{1}$ function such that $\left|u^{\prime}\right|^{p-2} u^{\prime} \in \mathcal{C}^{1}([0,+\infty))$.

We start with a local uniqueness and existence result which is analogous to a result due to Serrin and Veron (see [3], Theorem 5.2). 
On the radial solutions of a degenerate quasilinear elliptic equation in $\mathbb{R}^{N}$

Proposition 1.1. - Assume $q \geqslant p-1>1$. Then for any $\gamma \in \mathbb{R}^{*}$, there exists a constant $r_{\gamma}>0$ such that, problem $(P)$ admits a unique solution $u$ in $\left[0, r_{\gamma}\right)$.

Proof. - The proof is divided in three steps.

Step 1: Local uniqueness.

If $u$ is a solution of problem $(P)$ in $\left[0, r_{\gamma}\right)$. Then $u$ satisfies the following equation

$$
\left(r^{N-1}\left|u^{\prime}\right|^{p-2} u^{\prime}\right)^{\prime}+\frac{q+1-p}{p(q-1)} r^{N} u^{\prime}+r^{N-1} f(u)=0
$$

where $f$ is given in (0.8). Then

$$
u(r)=\gamma-\int_{0}^{r} G\left\{\frac{q+1-p}{p(q-1)} s u(s)+s^{1-N} \int_{0}^{s} \sigma^{N-1} F(u(\sigma)) d \sigma\right\} d s
$$

where

$$
G(s)=|s|^{-\frac{p-2}{p-1}} s
$$

and

$$
F(s)=\frac{1}{q-1}\left(1-\frac{N(q+1-p)}{p}\right) s+|s|^{q-1} s .
$$

Note that $F$ is a locally Lipschitz continuous function.

Let $v$ be another solution of $(P)$ in $\left[0, r_{\gamma}\right)$. Hence, for any $r \in\left(0, r_{\gamma}\right)$

$$
|u(r)-v(r)| \leqslant \int_{0}^{r}|G(A(s))-G(B(s))| d s
$$

where

and

$$
A(s)=\frac{q+1-p}{p(q-1)} s u(s)+s^{1-N} \int_{0}^{s} \sigma^{N-1} F(u(\sigma)) d \sigma
$$

$$
B(s)=\frac{q+1-p}{p(q-1)} s v(s)+s^{1-N} \int_{0}^{s} \sigma^{N-1} F(v(\sigma)) d \sigma
$$

for $0<s \leqslant r<r_{\gamma}$.

Next, let

$$
\phi(s)=\operatorname{Min}(|A(s)|,|B(s)|) .
$$

It is not difficult to see that

$$
|G(A(s))-G(B(s))| \leqslant \frac{G(\phi(s))}{\phi(s)}|A(s)-B(s)| .
$$


Substituting this last inequality into (1.5) we obtain

$$
|u(r)-v(r)| \leqslant \int_{0}^{r} \frac{G(\phi(s))}{\phi(s)}|A(s)-B(s)| d s .
$$

Let $m>0$ and $K_{m}$ be the Lipschitz constant of $F$ on $[\gamma-m, \gamma+m]$. In view of the continuity of $u$ and $v$, there exists $r_{0}>0$ such that

$$
\operatorname{Max}_{0 \leqslant r \leqslant r_{0}}(|u(r)-\gamma|,|v(r)-\gamma|) \leqslant m .
$$

Let

$$
\alpha=\frac{|\gamma|\left(\frac{1}{q-1}+|\gamma|^{q-1}\right)}{2\left(K_{m}+\frac{N(q+1-p)}{p(q-1)}\right)}
$$

Notice that, since

$$
K_{m} \leqslant \sup _{[\gamma-m, \gamma+m]}\left|F^{\prime}(s)\right| \leqslant \frac{1}{q-1}\left|1-\frac{N(q+1-p)}{p}\right|+q(|\gamma|+m)^{q-1},
$$

it is possible to choose $m$ such that $m \leqslant \alpha$. By using (1.11), we obtain easily the following inequalities on $\left[0, r_{0}\right]$

$$
\begin{gathered}
|F(u(s))-F(\gamma)| \leqslant K_{m}|u(s)-\gamma| \leqslant m K_{m} \\
\frac{F(\gamma)-m K_{m}}{N} s \leqslant s^{1-N} \int_{0}^{s} \sigma^{N-1} F(u(\sigma)) d \sigma \leqslant \frac{F(\gamma)+m K_{m}}{N} s
\end{gathered}
$$

and

$$
(\gamma-m) s \leqslant s u(s) \leqslant(\gamma+m) s .
$$

Combining (1.15)) and (1.16) we deduce that $A(s)$ has a constant sign on $\left[0, r_{0}\right]$. On the other hand, since $\gamma \neq 0$ we have

$$
\frac{q+1-p}{p(q-1)} \gamma+\frac{F(\gamma)}{N} \neq 0
$$

Moreover, if we set

$$
\beta=\frac{1}{2}\left|\frac{F(\gamma)}{N}+\frac{q+1-p}{p(q-1)} \gamma\right|=\frac{|\gamma|}{2 N}\left(\frac{1}{q-1}+|\gamma|^{q-1}\right)
$$

we deduce, from the choice of $m$, that

$$
|A(s)| \geqslant \beta s \quad \text { for } 0 \leqslant s \leqslant r \leqslant r_{0} .
$$


On the radial solutions of a degenerate quasilinear elliptic equation in $\mathbb{R}^{N}$

Of course the same estimate is also valid for $B(s)$. As a consequence

$$
\phi(s) \geqslant \beta s \quad \text { for any } 0 \leqslant s \leqslant r \leqslant r_{0} .
$$

This last inequality, together with (1.10) and the fact that the function $r \longrightarrow \frac{G(r)}{r}$ is decreasing implies that

$$
|u(r)-v(r)| \leqslant \int_{0}^{r} \frac{G(\beta s)}{\beta s}|A(s)-B(s)| d s,
$$

for every $r \in\left(0, r_{0}\right)$. But

$$
\begin{gathered}
|A(s)-B(s)| \leqslant \frac{q+1-p}{p(q-1)} s|u(s)-v(s)| \\
+K_{m} s^{1-N} \int_{0}^{s} \sigma^{N-1}|u(\sigma)-v(\sigma)| d \sigma .
\end{gathered}
$$

So that

We have

$$
\begin{aligned}
& |u(r)-v(r)| \leqslant \frac{q+1-p}{p(q-1)} \int_{0}^{r} \frac{G(\beta s)}{\beta s} s|u(s)-v(s)| d s \\
& \quad+K_{m} \int_{0}^{r} \frac{G(\beta s)}{\beta s} s^{1-N}\left(\int_{0}^{s} \sigma^{N-1}|u(\sigma)-v(\sigma)| d \sigma\right) d s .
\end{aligned}
$$

$$
\begin{aligned}
& K_{m} \int_{0}^{r} \frac{G(\beta s)}{\beta s} s^{1-N}\left(\int_{0}^{s} \sigma^{N-1}|u(\sigma)-v(\sigma)| d \sigma\right) d s \\
= & K_{m} \int_{0}^{r} \sigma^{N-1}|u(\sigma)-v(\sigma)|\left(\int_{\sigma}^{r} s^{1-N} \frac{G(\beta s)}{\beta s} d s\right) d \sigma .
\end{aligned}
$$

On the other hand

$$
\int_{\sigma}^{r} s^{1-N} \frac{G(\beta s)}{\beta s} d s \leqslant \frac{p-1}{N(p-1)-p} \beta^{-\frac{p-2}{p-1}} \sigma^{\frac{p}{p-1}-N} .
$$

Hence (1.23) implies that

$$
|u(r)-v(r)| \leqslant \int_{0}^{r}|u(s)-v(s)| a(s) d s, \quad \text { for } 0 \leqslant r \leqslant r_{0},
$$

where

$$
a(s)=\left(\frac{q+1-p}{p(q-1)}+\frac{p-1}{N(p-1)-p} K_{m}\right) \beta^{-\frac{p-2}{p-1}} s^{\frac{1}{p-1}} .
$$

Finally using Gronwall's lemma we conclude that $u=v$ on $\left[0, r_{0}\right]$. 
Step 2: Local existence

Let $m, K_{m}, \alpha$ and $\beta$ be the previously chosen constants. Set

$$
\delta=\frac{\frac{q+1-p}{p(q-1)}|\gamma|+\frac{|F(\gamma)|}{N}}{2 \frac{K_{m}}{N} \frac{p}{N(p-1)-p}}
$$

and

$$
m_{1}=\inf (m, \alpha, \delta)
$$

Let $I=\left[0, r_{\gamma}\right]$, where $r_{\gamma}$ is given by

$$
\begin{aligned}
r_{\gamma} & =\max \left\{r \geqslant 0: \int_{0}^{r} G(\beta s) d s \leqslant \frac{\beta m_{1}}{\frac{q+1-p}{p(q-1)}\left(|\gamma|+m_{1}\right)+\frac{|F(\gamma)|+m_{1} K_{m}}{N}}\right\} \\
& =\left(\frac{p}{p-1}\right)^{\frac{p-1}{p}}\left(\frac{\beta^{\frac{p-2}{p-1}} m_{1}}{\frac{q+1-p}{p(q-1)}\left(|\gamma|+m_{1}\right)+\frac{|F(\gamma)|+m_{1} K_{m}}{N}}\right)^{\frac{p-1}{p}} \cdot
\end{aligned}
$$

For the sequel, let $C(I, \mathbb{R})$ denote the Banach space of continuous functions on $I$ and consider the following complete metric space

$$
E_{\gamma}=\left\{\varphi \in C(I, \mathbb{R}):\|\varphi-\gamma\| \leqslant m_{1}\right\} .
$$

Define the mapping $T$ on $E_{\gamma}$, by

$$
T(\varphi)(r)=\gamma-\int_{0}^{r} G\left\{\frac{q+1-p}{p(q-1)} s \varphi(s)+s^{1-N} \int_{0}^{s} \sigma^{N-1} F(\varphi(\sigma)) d \sigma\right\} d s
$$

for $\varphi \in E_{\gamma}$ and $r \in\left[0, r_{\gamma}\right)$. Here $G$ and $F$ have the same meaning as in the previous formulas (1.3) and (1.4).

We claim that $T$ maps $E_{\gamma}$ into itself. To see this fix $\varphi \in E_{\gamma}$. Using (1.29) we can prove, as in step 1 , that $\frac{q+1-p}{p(q-1)} s \varphi(s)+s^{1-N} \int_{0}^{s} \sigma^{N-1} F(\varphi(\sigma)) d \sigma$ has a constant sign on $\left[0, r_{\gamma}\right)$ and consequently we have the following estimates

$$
\left|\frac{q+1-p}{p(q-1)} s \varphi(s)+s^{1-N} \int_{0}^{s} \sigma^{N-1} F(\varphi(\sigma)) d \sigma\right| \geqslant \beta s, \text { for } 0<s<r_{\gamma}
$$


On the radial solutions of a degenerate quasilinear elliptic equation in $\mathbb{R}^{N}$

and

$$
\begin{gathered}
\frac{q+1-p}{p(q-1)} s|\varphi(s)|+s^{1-N} \int_{0}^{s} \sigma^{N-1}|F(\varphi(\sigma))| d \sigma \leqslant \\
\left\{\frac{q+1-p}{p(q-1)}\left(|\gamma|+m_{1}\right)+\frac{|F(\gamma)|+m_{1} K_{m}}{N}\right\} s .
\end{gathered}
$$

Moreover

$$
\begin{gathered}
\left|G\left\{\frac{q+1-p}{p(q-1)} s \varphi(s)+s^{1-N} \int_{0}^{s} \sigma^{N-1} F(\varphi(\sigma)) d \sigma\right\}\right| \\
\leqslant \frac{G\left\{\frac{q+1-p}{p(q-1)} s \varphi(s)+s^{1-N} \int_{0}^{s} \sigma^{N-1} F(\varphi(\sigma)) d \sigma\right\}}{\frac{q+1-p}{p(q-1)} s \varphi(s)+s^{1-N} \int_{0}^{s} \sigma^{N-1}|F(\varphi(\sigma))| d \sigma} \\
\left\{\frac{q+1-p}{p(q-1)} s|\varphi(s)|+s^{1-N} \int_{0}^{s} \sigma^{N-1}|F(\varphi(\sigma))| d \sigma\right\} .
\end{gathered}
$$

From these estimates we also obtain

$$
|T(\varphi)(r)-\gamma| \leqslant \int_{0}^{r} \frac{G(\beta s)}{\beta s}\left\{\frac{q+1-p}{p(q-1)}\left(|\gamma|+m_{1}\right)+\frac{|F(\gamma)|+m_{1} K_{m}}{N}\right\} s d s .
$$

Then, from the choice of $r_{\gamma}$, we deduce that

$$
|T(\varphi)(r)-\gamma| \leqslant m_{1} ; \quad \text { for } \varphi \in E_{\gamma} \text { and } r \in\left(0, r_{\gamma}\right) \text {. }
$$

Now it is clear that $T$ is a contraction. In fact, as in step 1, we can show that for any $\varphi_{1}, \varphi_{2} \in E_{\gamma}$ and $r \in\left(0, r_{\gamma}\right)$

$$
\left|T\left(\varphi_{1}\right)(r)-T\left(\varphi_{2}\right)(r)\right| \leqslant \int_{0}^{r}\left|\varphi_{1}(s)-\varphi_{2}(s)\right| a(s) d s v
$$

where $\mathrm{a}$ is defined by (1.27).

Putting together (1.29) and (1.30), we get

$$
\int_{0}^{r_{\gamma}} a(s) d s<1
$$

The Banach contraction theorem implies that there exists a unique function solving the problem $(P)$ in $\left[0, r_{\gamma}\right)$. 
Step 3: $\left|u^{\prime}\right|^{p-2} u^{\prime} \in C^{1}\left(\left[0, r_{\gamma}\right)\right)$.

We have just to prove that $\left|u^{\prime}\right|^{p-2} u^{\prime}$ is a $C^{1}$ function near $r=0$. Integrating equation (1.1) from 0 to $r$, we obtain

$$
\begin{gathered}
\left|u^{\prime}\right|^{p-2} u^{\prime}(r)=-\frac{q+1-p}{p(q-1)} r u(r)- \\
r^{1-N} \int_{0}^{r} s^{N-1} u(s)\left\{\frac{1}{q-1}-\frac{N(q+1-p)}{p(q-1)}+|u|^{q-1}(s)\right\} d s
\end{gathered}
$$

which yields

$$
\lim _{r \rightarrow 0} \frac{\left|u^{\prime}\right|^{p-2} u^{\prime}(r)}{r}=-\frac{\gamma}{N}\left(\frac{1}{q-1}+|\gamma|^{q-1}\right) .
$$

On the other hand, since $u$ satisfies equation (0.6) then

$$
\lim _{r \rightarrow 0}\left(\left|u^{\prime}\right|^{p-2} u^{\prime}\right)^{\prime}(r)=-\frac{\gamma}{N}\left(\frac{1}{q-1}+|\gamma|^{q-1}\right) .
$$

Hence

$$
\left|u^{\prime}\right|^{p-2} u^{\prime} \in C^{1}\left(\left[0, r_{\gamma}\right)\right) \text {. }
$$

The proof is complete.

REMARK 1.1. - It is possible to see that for $N \geqslant 4$ and $q \geqslant p-1>1$ we have $\delta \geqslant \alpha$. Thus, in this case we can choose $m_{1}=\inf (m, \alpha)$ in the proof of the previous proposition.

Proposition 1.2. - For any $\gamma \in \mathbb{R}^{*}$, there exists a unique solution $u$ of problem $(P)$ in $[0,+\infty)$, which satisfies

$$
|u(r)| \leqslant|\gamma|, \quad \forall r \geqslant 0,
$$

and

$$
\left|u^{\prime}(r)\right| \leqslant\left(\frac{p}{p-1}\right)^{1 / p}\left(\frac{\gamma^{2}}{2(q-1)}+\frac{|\gamma|^{q+1}}{q+1}\right)^{1 / p}, \quad \forall r \geqslant 0 .
$$

Proof. - The proof is divided in two steps.

Step 1. Global existence. 
On the radial solutions of a degenerate quasilinear elliptic equation in $\mathbb{R}^{N}$

Thanks to proposition 1.1 , for any $\gamma \in \mathbb{R}_{*}^{+}$we know the local existence and uniqueness of a solution $u$ of problem $(P)$. In order to establish global existence, we define the energy function

$$
E(r)=\frac{p-1}{p}\left|u^{\prime}\right|^{p}(r)+\frac{|u|^{2}(r)}{2(q-1)}+\frac{|u|^{q+1}(r)}{q+1} .
$$

According to (1.1), $E$ satisfies that

$$
E^{\prime}(r)=-\left\{\frac{N-1}{r}\left|u^{\prime}\right|^{p}(r)+\frac{q+1-p}{p(q-1)} r\left|u^{\prime}\right|^{2}(r)\right\} \leqslant 0 .
$$

Hence $u$ and $u^{\prime}$ are bounded, and then $u$ exists for all $r \geqslant 0$.

Moreover, since $u^{\prime}(0)=0, E(r) \leqslant E(0)$ and the fact that the function $x \longrightarrow \frac{x^{2}}{2(q-1)}+\frac{|x|^{q+1}}{q+1}$ is increasing on $[0,+\infty)$, we get (1.45) and (1.46).

Step 2. Global uniqueness

Since the energy function $E$ is non increasing, we see that if $u=0$ and $u^{\prime}=0$ at some point $r_{0}>0$, then $u(r)=0$ for all $r \geqslant r_{0}$. Hence, we can suppose that $\left(u\left(r_{0}\right), u^{\prime}\left(r_{0}\right)\right) \neq(0,0)$ and the uniqueness is treated similarly as in proposition 1.1 .

REMARK 1.2. - It is not difficult to see that the solution $u$ of $(P)$ is a $\mathcal{C}^{\infty}$ function at any point $r>0$ whenever $u^{\prime}(r) \neq 0$.

\section{Asymptotic behaviour of solutions}

In this section we give some preliminary results for radial solutions of (0.1). Here and in the rest of the paper, we assume $N \geqslant 1, q>p-1>1$ and $\gamma>0$. Using equation (1.1) we immediately have the following lemma

LEMMA 2.1. - Suppose $u$ is a solution of $(P)$. Then any critical point $r$ of $u$ must be a relative minimum if $u(r)<0$ or a relative maximum if $u(r)>0$.

Now, proceeding as in [6], we shall prove the following result

LEMMA 2.2. - Suppose $u$ is a solution of $(P)$. Then the energy function $E(r)$ given by (1.49) goes to zero as $r \longrightarrow \infty$. 
Proof. - Since $E^{\prime}(r) \leqslant 0$ and $E(r) \geqslant 0$ for all $r>0$, then there is a constant $l \geqslant 0$ such that $\lim _{r \rightarrow \infty} E(r)=l$. Suppose for contradiction that $l>0$. Hence there exists $r_{1}>0$ such that

$$
E(r) \geqslant \frac{l}{2}, \quad \text { for all } r \geqslant r_{1} .
$$

Let us define disjoints subsets $I_{1}$ and $I_{2}$ of $\left[r_{1},+\infty\right)$ by

$$
I_{1}=\left\{r \geqslant r_{1}: \frac{u^{2}(r)}{2(q-1)}+\frac{|u|^{q+1}(r)}{q+1}<\frac{1}{4} l\right\}
$$

and

$$
I_{2}=\left\{r \geqslant r_{1}: \frac{u^{2}(r)}{2(q-1)}+\frac{|u|^{q+1}(r)}{q+1} \geqslant \frac{1}{4} l\right\} .
$$

Let $r \in\left[r_{1},+\infty[\right.$. We distinguish two cases.

(i) $r \in I_{1}$, from (2.1), we obtain

$$
\left|u^{\prime}\right|^{p}(r) \geqslant c_{0}=\frac{p}{4(p-1)} l .
$$

(ii) $r \in I_{2}$. Remembering that the function $x \rightarrow \frac{x^{2}}{2(q-1)}+\frac{|x|^{q+1}}{q+1}$ is increasing on $[0,+\infty)$, we obtain the existence of a positive constant $c_{1}$ such that $|u(r)| \geqslant c_{1}$. Thus, for any $r \in I_{2}$ we have

$$
\frac{u^{2}(r)}{q-1}+|u|^{q+1}(r) \geqslant c_{2}
$$

where $c_{2}=\frac{c_{1}^{2}}{q-1}+c_{1}^{q+1}$. Putting together (2.2) and (2.3) the following inequality holds for any $r \geqslant r_{1}$

$$
\left|u^{\prime}\right|^{p}(r)+\frac{u^{2}(r)}{q-1}+|u|^{q+1}(r) \geqslant c=\min \left\{c_{0}, c_{2}\right\} .
$$

Now consider the function

$$
\begin{gathered}
D(r)=E(r)+\frac{N-1}{2 r}\left|u^{\prime}\right|^{p-2} u^{\prime} u(r)+\frac{N-1}{4} \frac{q+1-p}{p(q-1)}|u|^{2}(r)+ \\
+\frac{q+1-p}{p(q-1)} \int_{0}^{r} s\left|u^{\prime}\right|^{2}(s) d s . \\
-422-
\end{gathered}
$$


On the radial solutions of a degenerate quasilinear elliptic equation in $\mathbb{R}^{N}$

Applying equation (0.6), we obtain the following equality

$$
D^{\prime}(r)=-\frac{N-1}{2 r}\left\{\left|u^{\prime}\right|^{p}(r)+\frac{u^{2}(r)}{q-1}+|u|^{q+1}(r)+\frac{N}{r}\left|u^{\prime}\right|^{p-2} u^{\prime} u(r)\right\} \text {. }
$$

Recall that $u$ and $u^{\prime}$ are bounded, so that there exists $r_{2}\left(\geqslant r_{1}\right)$ such that

$$
\frac{N}{r}\left|u^{\prime}\right|^{p-2} u^{\prime} u(r) \geqslant-\frac{c}{2}, \text { for } r \geqslant r_{2}
$$

Combining (2.4) and (2.7) yields

$$
\left|u^{\prime}\right|^{p}(r)+\frac{u^{2}(r)}{q-1}+|u|^{q+1}(r)+\frac{N}{r}\left|u^{\prime}\right|^{p-2} u^{\prime} u(r) \geqslant \frac{c}{2}, \text { for } r \geqslant r_{2} .
$$

Integrating equation (2.6) between $r_{2}$ and $r$ we get

$$
D(r) \leqslant D\left(r_{2}\right)-\frac{c(N-1)}{4} \log \frac{r}{r_{2}}, \quad \text { for } r \geqslant r_{2} .
$$

But, $E(r)+\frac{N-1}{2 r}\left|u^{\prime}\right|^{p-2} u^{\prime} u(r) \leqslant D(r)$, and using again the boundedness of $u$ and $u^{\prime}$, we get from (2.9) that $\lim _{r \rightarrow+\infty} E(r)=-\infty$. This is impossible. Hence $l=0$ and the proof is ended . $\square$

As a consequence of the above lemma, we derive the following result

Corollary 2.1. - Suppose $u$ is a solution of $(P)$. Then necessarily $u(r)$ and $u^{\prime}(r)$ go to 0 as $r \longrightarrow+\infty$.

\section{Strictly positive solutions}

The goal of this section is to investigate the existence of strictly positive solutions of $(P)$. But first of all, we establish some results on the behavior of such solutions.

PROPOSITION 3.1. - If $u$ is a strictly positive solution of $(P)$ then

$$
u^{\prime}(r)<0, \quad \text { for all } r>0 .
$$

Proof. - Suppose for contradiction that there exists $r_{0}>0$ such that $u^{\prime}\left(r_{0}\right) \geqslant 0$. As

$$
\left(\left|u^{\prime}\right|^{p-2} u^{\prime}\right)^{\prime}(0)=-\frac{\gamma}{N}\left(\frac{1}{q-1}+\gamma^{q-1}\right)<0
$$


then there exists $r_{1} \in\left(0, r_{0}\right]$ such that $u\left(r_{1}\right)>0, u^{\prime}\left(r_{1}\right)=0$ and $\left(\left|u^{\prime}\right|^{p-2} u^{\prime}\right)^{\prime}\left(r_{1}\right) \geqslant 0$. This contradicts the fact that

$$
\begin{gathered}
\left(\left|u^{\prime}\right|^{p-2} u^{\prime}\right)^{\prime}\left(r_{1}\right)+\frac{N-1}{r_{1}}\left|u^{\prime}\right|^{p-2} u^{\prime}\left(r_{1}\right)+\frac{q+1-p}{p(q-1)} r_{1} u^{\prime}\left(r_{1}\right) \\
+\frac{u\left(r_{1}\right)}{q-1}+u^{q}\left(r_{1}\right)=0 .
\end{gathered}
$$

Thus, $u^{\prime}(r)<0$, for all $r>0$.

Proposition 3.2. - Let $u$ be a strictly positive solution of $(P)$. Then there exists $r_{0}>0$ such that the function $\frac{u^{\prime}(r)}{u(r)}$ is monotone in $\left[r_{0},+\infty\right)$.

As a consequence of this result, we have

COROLlaRY 3.1. - Let $u$ be a strictly positive solution of $(P)$. Then $\lim _{r \rightarrow \infty} \frac{u^{\prime}(r)}{u(r)}$ exists in $[-\infty, 0]$.

One of the main tools for the proof of proposition 3.2. is the following lemma.

Lemma 3.1. - Let $u$ be a strictly positive solution of $(P)$. Then there exists $r_{1}>0$ such that

$$
a^{\prime}(r) u^{\prime}(r)+b^{\prime}(r) u(r)<0
$$

for any $r>r_{1}$ satisfying

$$
\left(u^{\prime}\right)^{2}(r)+a(r) u(r) u^{\prime}(r)+b(r) u^{2}(r)=0
$$

where

$$
a(r)=\frac{1}{p-1}\left(\frac{N-1}{r}+\frac{q+1-p}{p(q-1)} \frac{r}{\left|u^{\prime}\right|^{p-2}(r)}\right)
$$

and

$$
\begin{gathered}
b(r)=\frac{1}{(p-1)\left|u^{\prime}\right|^{p-2}(r)}\left(\frac{1}{q-1}+u^{q-1}(r)\right) . \\
-424-
\end{gathered}
$$


On the radial solutions of a degenerate quasilinear elliptic equation in $\mathbb{R}^{N}$

Proof. - Proposition 3.1 together with Remark 1.2 and equation (0.6) imply that $u$ satisfies the following equation

$$
u^{\prime \prime}(r)+a(r) u^{\prime}(r)+b(r) u(r)=0, \text { for } \quad r>0 .
$$

Define the set

$$
J_{u}=\left\{r>0:\left(u^{\prime}\right)^{2}(r)+a(r) u(r) u^{\prime}(r)+b(r) u^{2}(r)=0\right\} .
$$

It is clear that for any $r \in J_{u}$, the following equations hold

$$
u u^{\prime \prime}=\left(u^{\prime}\right)^{2}
$$

and

$$
\frac{u}{(p-1)\left|u^{\prime}\right|^{p-2}}\left\{\frac{q+1-p}{p(q-1)} r u^{\prime}+\frac{u}{q-1}+u^{q}\right\}=-\left(u^{\prime}\right)^{2}-\frac{N-1}{(p-1)} \frac{u u^{\prime}}{r} .
$$

This leads to

$$
\begin{aligned}
& (p-1)\left(a^{\prime} u^{\prime}+b^{\prime} u\right)=\left|u^{\prime}\right|\left\{\frac{N-1}{r^{2}}+(p-2)(N-1) \frac{\left|u^{\prime}\right|}{r u}-\right. \\
& \left.(p-1)(p-2) \frac{\left|u^{\prime}\right|^{2}}{u^{2}}-\frac{q+1-p}{p(q-1)} \frac{1}{\left|u^{\prime}\right|^{p-2}}-(q-1) \frac{u^{q-1}}{\left|u^{\prime}\right|^{p-2}}\right\} .
\end{aligned}
$$

for any $r \in J_{u}$.

On the other hand equation (3.11) is equivalent to

$$
\begin{aligned}
u^{2}\left(\frac{1}{q-1}+u^{q-1}\right)+ & \frac{q+1-p}{p(q-1)} r u u^{\prime}+(p-1)\left|u^{\prime}\right|^{p} \\
& +\frac{N-1}{r}\left|u^{\prime}\right|^{p-2} u^{\prime} u=0 .
\end{aligned}
$$

Set

$$
A(r)=\frac{N-1}{r}\left|u^{\prime}\right|^{p-2}(r)+\frac{q+1-p}{p(q-1)} r
$$

and

$$
B(r)=\frac{1}{q-1}+u^{q-1}(r) .
$$


Then, $u$ satisfies

$$
B u^{2}-A\left|u^{\prime}\right| u+(p-1)\left|u^{\prime}\right|^{p}=0, \text { on } J_{u} .
$$

so that

$$
u=\frac{A \pm \sqrt{A^{2}-4(p-1) B\left|u^{\prime}\right|^{p-2}}}{2 B}\left|u^{\prime}\right|, \quad \text { on } J_{u} .
$$

Set

$$
J_{u}^{ \pm}=\left\{r>0: u(r)=\frac{A \pm \sqrt{A^{2}-4(p-1) B\left|u^{\prime}\right|^{p-2}}}{2 B}\left|u^{\prime}\right|(r)\right\} .
$$

It is clear that $J_{u}=J_{u}^{+} \cup J_{u}^{-}$.

First, observe that from corollary 2.1, we have

$$
A^{2}(r)>>4(p-1) B\left|u^{\prime}\right|^{p-2}(r), \text { for large } r \text {. }
$$

Next, we consider two cases.

(i) $r \in J_{u}^{+}$is large enough. Then we have the following behaviour

$$
u(r) \sim \frac{q+1-p}{p} r\left|u^{\prime}\right|(r) .
$$

Carrying this last result in (3.12), we obtain

$$
\begin{aligned}
&(p-1)\left(a^{\prime} u^{\prime}+b^{\prime} u\right) \sim\left|u^{\prime}\right|\left\{\frac{N-1}{r^{2}}+(N-1)(p-2) \frac{p}{(q+1-p) r^{2}}\right. \\
&\left.-(q-1) \frac{u^{q-1}}{\left|u^{\prime}\right|^{p-2}}-\frac{p^{2}(p-1)(p-2)}{(q+1-p)^{2} r^{2}}-\frac{q+1-p}{p(q-1)\left|u^{\prime}\right|^{p-3}}\right\} .
\end{aligned}
$$

Using again corollary 2.1 , we deduce that

$$
(p-1)\left(a^{\prime} u^{\prime}+b^{\prime} u\right) \sim-\frac{q+1-p}{p(q-1)} \frac{1}{\left|u^{\prime}\right|^{p-2}}<0 .
$$

(ii) $r \in J_{u}^{-}$is large enough, then

$$
\begin{gathered}
u(r) \sim \frac{p(p-1)(q-1)}{q+1-p} \frac{\left|u^{\prime}\right|^{p-1}}{r} . \\
-426-
\end{gathered}
$$


On the radial solutions of a degenerate quasilinear elliptic equation in $\mathbb{R}^{N}$

Hence, from (3.12), we get

$$
(p-1)\left(a^{\prime} u^{\prime}+b^{\prime} u\right) \sim-\frac{(p-2)(q+1-p)^{2}}{p^{2}(p-1)(q-1)^{2}} \frac{r^{2}}{\left|u^{\prime}\right|^{2 p-3}}<0 .
$$

So (3.4) holds and the proof is ended .

Proof of Proposition 3.2. - Introducing the functions

$$
\tilde{a}(r)=a(r)+k(r) u(r), \quad \text { for } r>0
$$

and

$$
\widetilde{b}(r)=b(r)-k(r) u^{\prime}(r), \quad \text { for } r>0
$$

where $a(r)$ and $b(r)$ are given in lemma 3.1, and function $k(r)$ will be chosen later. As $u$ satisfies (3.8), we have

$$
u^{\prime \prime}+\tilde{a} u^{\prime}+\tilde{b} u=0 .
$$

Let $w=u^{\prime}$, then $w$ satisfies

$$
w^{\prime \prime}+\widetilde{a} w^{\prime}+\widetilde{b} w=-\left(a^{\prime} u^{\prime}+b^{\prime} u\right)-k\left[\left(u^{\prime}\right)^{2}+a u u^{\prime}+b u^{2}\right] .
$$

Applying lemma 3.1, it is possible to choose $r_{1}>0$ and $k(r)$ such that

$$
-\left(a^{\prime} u^{\prime}+b^{\prime} u\right)-k\left[\left(u^{\prime}\right)^{2}+a u u^{\prime}+b u^{2}\right]>0 \text {, for } r>r_{1} .
$$

Therefore

$$
w^{\prime \prime}+\tilde{a} w^{\prime}+\tilde{b} w>0, \quad \text { on }\left(r_{1},+\infty\right) .
$$

If we put $y=\frac{w}{u}=\frac{u^{\prime}}{u}$, then we obtain

$$
y^{\prime \prime}+\left(\widetilde{a}+2 \frac{u^{\prime}}{u}\right) y^{\prime}>0, \text { on }\left(r_{1},+\infty\right) .
$$

So, we have either $y^{\prime}(r)<0$, for all $r>r_{1}$ or there exists $r_{2} \geqslant r_{1}$ such that $y^{\prime}(r)>0$, for all $r>r_{2}$, which ends the proof.

In the next two propositions, we will give more precise asymptotic behavior for strictly positive solutions.

Proposition 3.3. - Let $u$ be a strictly positive solution of $(P)$ then

$$
\lim _{r \rightarrow+\infty} \frac{r u^{\prime}(r)}{u(r)}=-\frac{p}{q+1-p} .
$$


Proof. - Set $L=\lim _{r \rightarrow+\infty} \frac{u^{\prime}(r)}{u(r)}$. Thanks to corollary 3.1, we have $L \in$ $[-\infty, 0]$. We claim that $L \neq-\infty$. Indeed, suppose that $L=-\infty$. As we have $u(r)>0$ and $u^{\prime}(r)<0$, for all $r>0$, equation (0.6) is equivalent to

$$
\begin{aligned}
(p-1)\left|u^{\prime}\right|^{p-2} & \frac{u^{\prime \prime}}{r u^{\prime}}+\frac{N-1}{r^{2}}\left|u^{\prime}\right|^{p-2}+\frac{q+1-p}{p(q-1)} \\
& +\frac{u}{r u^{\prime}}\left(\frac{1}{q-1}+u^{q-1}\right)=0, \text { for } r>0 .
\end{aligned}
$$

Therefore, since $u$ and $u^{\prime}$ are bounded, by letting $r \longrightarrow+\infty$

$$
\lim _{r \rightarrow+\infty} u^{\prime \prime} \frac{\left|u^{\prime}\right|^{p-3}}{r}(r)=\frac{q+1-p}{p(p-1)(q-1)}
$$

we deduce that, for large $r$, we have

$$
\frac{1}{(p-2)}\left(\left|u^{\prime}\right|^{p-2}\right)^{\prime}(r)=-\frac{q+1-p}{p(p-1)(q-1)} r+O(r)
$$

which contradicts the fact that $\lim _{r \rightarrow+\infty} u^{\prime}(r)=0$.

We turn now to prove (3.32). By l'Hôpital's rule, we get

$$
L=\lim _{r \rightarrow+\infty} \frac{u^{\prime}(r)}{u(r)}=\lim _{r \rightarrow+\infty} \frac{u^{\prime \prime}(r)}{u^{\prime}(r)} .
$$

On the other hand, dividing equation ( 0.6$)$ by $u(r)$, we obtain the following equality

$$
\begin{aligned}
(p-1) \frac{\left|u^{\prime}\right|^{p-2} u^{\prime \prime}}{u}+ & \frac{N-1}{r} \frac{\left|u^{\prime}\right|^{p-2} u^{\prime}}{u}+\frac{q+1-p}{p(q-1)} \frac{r u^{\prime}}{u} \\
& +\left(\frac{1}{q-1}+u^{q-1}\right)=0, \quad \text { for } r>0 .
\end{aligned}
$$

Letting $r \longrightarrow+\infty$; the proposition follows by (3.36) and corollary 2.1 .

CoROllaRY 3.2. - Let $u$ be a strictly positive solution of $(P)$. Then, $\lim _{r \rightarrow+\infty} r^{\frac{p}{q+1-p}} u(r)$ exists and is a positive number. 
On the radial solutions of a degenerate quasilinear elliptic equation in $\mathbb{R}^{N}$

REMARK 3.1.- By using the previous result, we deduce that if there exist two constants $C$ and $m$ such that $u(r) \leqslant C r^{-m}$, for large $r$. Then $\left|u^{\prime}(r)\right| \leqslant \widetilde{C} r^{-m-1}$, for large $r$, where $\widetilde{C}$ is another constant.

Proposition 3.4. - Let $u$ be a strictly positive solution of $(P)$ such that $\lim _{r \rightarrow+\infty} r^{\frac{p}{q+1-p}} u(r)=0$. Then, for all $m>0$, we have $\lim _{r \rightarrow+\infty} r^{m} u(r)=0$ and $\lim _{r \rightarrow+\infty} r^{m+1} u^{\prime}(r)=0$.

Proof. - Let $\lambda=\frac{p}{q+1-p}$. Multiplying equation (0.6) by $r^{\lambda-1}$, then the following identity holds

$$
\begin{aligned}
& \left(r^{\lambda-1}\left|u^{\prime}\right|^{p-2} u^{\prime}\right)^{\prime}+(N-\lambda) r^{\lambda-2}\left|u^{\prime}\right|^{p-2} u^{\prime}+ \\
& \frac{q+1-p}{p(q-1)}\left(r^{\lambda} u\right)^{\prime}+r^{\lambda-1} u^{q}=0, \text { for } r>0 .
\end{aligned}
$$

Integrating from $x$ to $y \quad(0<x<y<+\infty)$, we get

$$
\begin{gathered}
y^{\lambda-1}\left|u^{\prime}\right|^{p-2} u^{\prime}(y)-x^{\lambda-1}\left|u^{\prime}\right|^{p-2} u^{\prime}(x)+(N-\lambda) \int_{x}^{y} r^{\lambda-2}\left|u^{\prime}\right|^{p-2} u^{\prime}(r) d r \\
+\frac{q+1-p}{p(q-1)} y^{\lambda} u(y)-\frac{q+1-p}{p(q-1)} x^{\lambda} u(x)+\int_{x}^{y} r^{\lambda-1} u^{q}(r) d r=0 .
\end{gathered}
$$

Letting $y \longrightarrow+\infty$, we obtain

$$
\begin{array}{r}
x^{\lambda-1}\left|u^{\prime}\right|^{p-2} u^{\prime}(x)+\frac{q+1-p}{p(q-1)} x^{\lambda} u(x)=\int_{x}^{+\infty} r^{\lambda-1} u^{q}(r) d r \\
+(N-\lambda) \int_{x}^{+\infty} r^{\lambda-2}\left|u^{\prime}\right|^{p-2} u^{\prime}(r) d r .
\end{array}
$$

Thus

$$
\begin{aligned}
\frac{q+1-p}{p(q-1)} x^{\lambda} u(x) \leqslant x^{\lambda-1}\left|u^{\prime}\right|^{p-1}(x)+\int_{x}^{+\infty} r^{\lambda-1} u^{q}(r) d r & \\
& +|N-\lambda| \int_{x}^{+\infty} r^{\lambda-2}\left|u^{\prime}\right|^{p-1}(r) d r . \\
& -429-
\end{aligned}
$$


It follows that if $u(x) \leqslant C x^{-m_{1}}$, (with $m_{1} \geqslant \lambda$ ), for large $x$ (consequently $\left|u^{\prime}(x)\right| \leqslant C x^{-m_{1}-1}$, by Remark 3.1) and we get

$$
\begin{gathered}
\int_{x}^{+\infty} r^{\lambda-1} u^{q}(r) d r \leqslant C x^{\lambda-m_{1} q} \\
x^{\lambda-1}\left|u^{\prime}\right|^{p-1}(x) \leqslant C x^{\lambda-1-(p-1)\left(m_{1}+1\right)}
\end{gathered}
$$

and

$$
\int_{x}^{+\infty} r^{\lambda-2}\left|u^{\prime}\right|^{p-1}(r) d r \leqslant C x^{\lambda-1-(p-1)\left(m_{1}+1\right)}
$$

where we denote by $C$ different constants.

Plugging all these estimates in (3.41), we deduce that

$$
u(x) \leqslant C\left(x^{-m_{1} q}+x^{-(p-1)\left(m_{1}+1\right)-1}\right), \text { for large } x .
$$

Then

$$
u(x) \leqslant C x^{-m_{2}}, \text { for large } x
$$

where

$$
m_{2}=\min \left(m_{1} q,(p-1)\left(m_{1}+1\right)+1\right) .
$$

Since $q>1$ and $p>2$, we have $m_{2}>m_{1}$. The proposition follows by induction starting with $m_{1}=\lambda$ and setting $m_{j+1}=\min \left(m_{j} q,\left(m_{j}+1\right)(p-\right.$ 1) +1$)$.

We may now turn to the main result of this section: the existence of strictly positive solutions of $(P)$ for sufficiently small initial values $\gamma$ and under suitable hypothesis on $p, q$ and $N$. More precisely, we have

Proposition 3.5. - Assume $q>\frac{p}{N}+p-1$ and $0<\gamma<\left(\frac{N(q+1-p)}{p(q-1)}-\frac{1}{q-1}\right)^{\frac{1}{q-1}}$. Then the solution $u$ of $(P)$ is strictly positive and $\lim _{r \rightarrow+\infty} r^{\frac{p}{q+1-p}} u(r)>0$.

Proof. - Suppose $u$ is not strictly positive and let $r_{0}$ be the first positive zero of $u$. Then, $u^{\prime}\left(r_{0}\right) \leqslant 0$ and $u(r)>0$, for all $r \in\left[0, r_{0}\right)$. Integrating equation (1.1) from 0 to $r_{0}$ gives the following formula

$$
r_{0}^{N-1}\left|u^{\prime}\right|^{p-2} u^{\prime}\left(r_{0}\right)=\int_{0}^{r_{0}} s^{N-1} u(s)\left[\frac{N(q+1-p)}{p(q-1)}-\frac{1}{q-1}-u^{q-1}(s)\right] d s .
$$


On the radial solutions of a degenerate quasilinear elliptic equation in $\mathbb{R}^{N}$

Since $|u(s)|<\gamma$ and from the choice of $\gamma$, we deduce that the right side of (3.48) is strictly positive but the left side is negative. This contradiction allows to confirm that $u$ is strictly positive and so $\lim _{r \rightarrow+\infty} r^{\frac{p}{q+1-p}} u(r) \geqslant 0$.

To finish the proof, one suppose, that $\lim _{r \rightarrow+\infty} r^{\frac{p}{q+1-p}} u(r)=0$. We have, for any $r>0$,

$$
\begin{aligned}
& r^{N-1}\left|u^{\prime}\right|^{p-2} u^{\prime}(r)+\frac{q+1-p}{p(q-1)} r^{N} u(r)= \\
& \int_{0}^{r} s^{N-1} u(s)\left[\frac{N(q+1-p)}{p(q-1)}-\frac{1}{q-1}-u^{q-1}(s)\right] d s .
\end{aligned}
$$

By using proposition 3.4, the left side of the last equality converges to zero as $r \longrightarrow+\infty$. But the right side is strictly positive. Then we have a contradiction and this completes the proof.

Proposition 3.6. - Assume $2<p<N$ and $q \geqslant \frac{N p}{N-p}-1$. Then for any $\gamma \in \mathbb{R}_{*}^{+}$, the solution $u(r, \gamma)$ of $(P)$ is strictly positive and

$$
\lim _{r \rightarrow+\infty} r^{\frac{p}{q+1-p}} u(r, \gamma)>0
$$

Proof. - (i) Assume that there exists some positive real $r$ such that $u(r)=0$. Setting

$$
R=\sup \{r>0: u>0 \text { on }[0, r[\} .
$$

Then $u$ is a radial solution of the following problem:

$$
\begin{cases}\Delta_{p} u+\frac{q+1-p}{p(q-1)} x \nabla u+\frac{u}{q-1}+u^{q}=0 & \text { in } B_{R} \\ u=0 & \text { on } \partial B_{R}\end{cases}
$$

where $B_{R}$ is the ball of radius $R$ centered at $x=0$.

Multiplying (3.51) by $u$, integrating over $B_{R}$ and using Green's formula and (3.52), we get

$$
\begin{gathered}
\int_{B_{R}}|\nabla u|^{p} d x=-\frac{N(q+1-p)}{2 p(q-1)} \int_{B_{R}} u^{2} d x \\
+\frac{1}{q-1} \int_{B_{R}} u^{2} d x+\int_{B_{R}} u^{q+1} d x \\
-431-
\end{gathered}
$$


Next, multiply (3.51) by $x . \nabla u$, we obtain

$$
\begin{aligned}
\int_{B_{R}}\left(\Delta_{p} u\right)(x . \nabla u) d x+\frac{q+1-p}{p(q-1)} \int_{B_{R}}(x \cdot \nabla u)^{2} d x \\
+\frac{1}{q-1} \int_{B_{R}} u(x \cdot \nabla u) d x+\int_{B_{R}} u^{q}(x \cdot \nabla u) d x=0 .
\end{aligned}
$$

To estimate the first integral we use Gauss-Ostrogradskii's formula to conclude that

$$
\begin{aligned}
& \int_{B_{R}}\left(\Delta_{p} u\right)(x . \nabla u)=-\int_{B_{R}}|\nabla u|^{p}-\frac{1}{p} \int_{B_{R}} x \cdot \nabla\left(|\nabla u|^{p}\right) d x \\
&+ \int_{\partial B_{R}}|\nabla u|^{p-2}(x . \nabla u) \nabla u . \nu d \sigma \\
&=\left(\frac{N}{p}-1\right) \int_{B_{R}}|\nabla u|^{p} d x+\left(1-\frac{1}{p}\right) \int_{\partial B_{R}}|\nabla u|^{q}(x . \nu) d \sigma .
\end{aligned}
$$

By standard computations we have

$$
\int_{B_{R}} u(x . \nabla u) d x=-\frac{N}{2} \int_{B_{R}} u^{2} d x
$$

and

$$
\int_{B_{R}} u^{q}(x . \nabla u) d x=-\frac{N}{q+1} \int_{B_{R}} u^{q+1} d x .
$$

Now let $k$ be a positive real such that

$$
k(N-k) \geqslant \frac{N(N-p)}{2 p}\left(1+\frac{N(p-2)}{p}\right) .
$$

If we define the operator $G$ by

$$
G u=k u+x \cdot \nabla u
$$

we obtain

$$
\int_{B_{R}}(x \nabla u)^{2} d x=\int_{B_{R}}(G u)^{2} d x+k(N-k) \int_{B_{R}} u^{2} d x .
$$

Plugging all these estimates in (3.54), we obtain

$$
\begin{aligned}
\left(\frac{N}{p}-1\right) \int_{B_{R}}|\nabla u|^{p} d x+ & \left(1-\frac{1}{p}\right) \int_{\partial B_{R}}|\nabla u|^{p}(x . \nu) d \sigma+ \\
& -432-
\end{aligned}
$$


On the radial solutions of a degenerate quasilinear elliptic equation in $\mathbb{R}^{N}$

$$
\begin{gathered}
\frac{1}{q-1}\left(\frac{q+1-p}{p} k(N-k)-\frac{N}{2}\right) \int_{B_{R}} u^{2} d x-\frac{N}{q+1} \int_{B_{R}} u^{q+1} d x \\
+\frac{q+1-p}{p(q-1)} \int_{B_{R}} G^{2}(u) d x=0 .
\end{gathered}
$$

Substituting (3.53) in this last equality we obtain

$$
\begin{gathered}
\left(\frac{N}{p}-1-\frac{N}{q+1}\right) \int_{B_{R}} u^{q+1} d x+\frac{q+1-p}{p(q-1)} \int_{B_{R}} G^{2}(u) d x \\
\quad+\left(1-\frac{1}{p}\right) \int_{\partial B_{R}}|\nabla u|^{p}(x . \nu) d \sigma+ \\
\left\{\left(\frac{N}{p}-1-\frac{N}{2}\right) \frac{1}{q-1}+\frac{q+1-p}{p(q-1)}\left(k(N-k)-\frac{N}{2}\right)\right\} \int_{B_{R}} u^{2} d x=0 .
\end{gathered}
$$

From the choice of $k$, the coefficient in front of the integral $\int_{B_{R}} u^{2} d x$ is strictly positive. On the other hand as $q \geqslant \frac{N P}{N-p}-1, \frac{N}{p}-1-\frac{N}{q+1} \geqslant 0$.

We deduce a contradiction with (3.62). Hence $u(r, \gamma)>0$ for any $r>0$.

(ii) Assume that $\lim _{r \rightarrow+\infty} r^{\frac{p}{q+1-p}} u(r)=0$ we deduce from proposition 3.4. that $\lim _{r \rightarrow+\infty} r^{m} u(r)=\lim _{r \rightarrow+\infty} r^{m+1} u^{\prime}(r)=0$ for any $m$.

Then we can let $R$ to the infinity in (3.62). As in step (i) we get a contradiction and the proof is complete.

\section{Non positive solutions}

In this section we investigate the existence and some properties of non positive solutions of $(P)$. Set $r_{0}=\inf \{r>0: u(r)=0\}$, the first positive zero of $u$; when $0<r_{0}<\infty, u$ is called nonpositive solution.

Proposition 4.1. - Assume $p-1<q \leqslant \frac{p}{N}+p-1$. Then there are no positive solutions of $(P)$.

Proof. - Suppose by contrary that $u$ is a positive solution of $(P)$. Then, for any $r>0$, the following equality holds true

$$
r^{N-1}\left|u^{\prime}\right|^{p-2} u^{\prime}(r)+\frac{q+1-p}{p(q-1)} r^{N} u(r)=
$$


Betteoui Benyounes, Abdelilah Gmira

$$
\frac{1}{q-1}\left(\frac{N(q+1-p)}{p}-1\right) \int_{0}^{r} s^{N-1} u(s) d s-\int_{0}^{r} s^{N-1} u^{q}(s) d s .
$$

This implies

$$
r^{N-1}\left|u^{\prime}\right|^{p-2} u^{\prime}(r)+\frac{q+1-p}{p(q-1)} r^{N} u(r) \leqslant 0, \quad \text { for all } r>0 .
$$

which means that

$$
u^{\prime}(r) \leqslant-\left(\frac{q+1-p}{p(q-1)}\right)^{\frac{1}{p-1}} r^{\frac{1}{p-1}} u^{\frac{1}{p-1}}(r), \quad r>0 .
$$

Integrating from 0 to $r$, we obtain

$$
u^{\frac{p-2}{p-1}}(r) \leqslant u^{\frac{p-2}{p-1}}(0)-\frac{p-2}{p}\left(\frac{q+1-p}{p(q-1)}\right)^{\frac{1}{p-1}} r^{\frac{p}{p-1}} .
$$

By letting $r \longrightarrow+\infty$, we get a contradiction.

Proposition 4.2. - Assume $2<p<N$ and $p-1<q<\frac{N p}{N-p}-1$. Then there exists $\gamma^{*}>0$ such that for any $\gamma>\gamma^{*}$ the solution $u$ of $(P)$ is non positive.

This proposition is a simple consequence of the previous lemma.

Lemma 4.1. - Assume $2<p<N$ and $p-1<q<\frac{N p}{N-p}-1$. Then there is no positive radial solution of equation

$$
\triangle_{p} u+u^{q}=0, \quad \text { in } \mathbb{R}^{N} .
$$

The proof of this lemma is given in [1]

LEMMA 4.2.- If for any $\gamma>0$ the solution $u$ of $(P)$ is strictly positive, then there exists a $\mathcal{C}^{1}$ function $v$ which satisfies

$$
\begin{aligned}
& \left(\left|v^{\prime}\right|^{p-2} v^{\prime}\right)^{\prime}+\frac{N-1}{r}\left|v^{\prime}\right|^{p-2} v^{\prime}+|v|^{q-1} v=0, \quad r>0 \\
& v(0)=1, \quad v^{\prime}(0)=0 \\
& v(r)>0, \quad \text { for all } r \geqslant 0
\end{aligned}
$$


On the radial solutions of a degenerate quasilinear elliptic equation in $\mathbb{R}^{N}$

Proof. - Let $k>0$ and $u_{k}$ be a solution of $(0.6)$ with $u_{k}(0)=k^{\frac{p}{q+1-p}}$ and $u_{k}^{\prime}(0)=0$. Define the function $v_{k}$ by

$$
v_{k}(r)=k^{-\frac{p}{q+1-p}} u_{k}\left(\frac{r}{k}\right), \quad \text { for } r \geqslant 0 .
$$

we have $v_{k} \in \mathcal{C}^{1}([0,+\infty))$ and $\left|v_{k}^{\prime}\right|^{p-2} v_{k}^{\prime} \in \mathcal{C}^{1}([0,+\infty))$. Some elementary computation show that $v_{k}$ satisfies

$$
\left\{\begin{aligned}
\left(\left|v_{k}^{\prime}\right|^{p-2} v_{k}^{\prime}\right)^{\prime} & +\frac{N-1}{r}\left|v_{k}^{\prime}\right|^{p-2} v_{k}^{\prime}+\frac{q+1-p}{p(q-1)} k^{-\frac{p(q-1)}{q+1-p}} r v_{k}^{\prime} \\
& +\frac{1}{q-1} k^{-\frac{p(q-1)}{q+1-p}} v_{k}+\left|v_{k}\right|^{q-1} v_{k}=0, \quad r>0
\end{aligned}\right.
$$

On the other hand, since

$$
\left|u_{k}(r)\right| \leqslant k^{\frac{p}{q+1-p}}, \quad \text { for all } r \geqslant 0
$$

we obtain

$$
\left|v_{k}(r)\right| \leqslant 1, \quad \text { for all } r \geqslant 0
$$

Now observe that $(4.10)$ is equivalent to

$$
\begin{gathered}
r^{N-1}\left|v_{k}^{\prime}\right|^{p-2} v_{k}^{\prime}(r)=-\frac{q+1-p}{p(q-1)} k^{-\frac{p(q-1)}{q+1-p}} r^{N} v_{k}(r)-\int_{0}^{r} s^{N-1}\left|v_{k}\right|^{q-1} v_{k}(s) d s \\
+\left(\frac{N(q+1-p)}{p(q-1)}-\frac{1}{q-1}\right) k^{-\frac{p(q-1)}{q+1-p}} \int_{0}^{r} s^{N-1} v_{k}(s) d s
\end{gathered}
$$

which implies, by using (4.12), that

$$
\begin{aligned}
r^{N-1}\left|v_{k}^{\prime}\right|^{p-1}(r) \leqslant & \frac{q+1-p}{p(q-1)} r^{N} k^{-\frac{p(q-1)}{q+1-p}}+\frac{r^{N}}{N} \\
& +\frac{1}{q-1}\left|\frac{N(q+1-p)}{p}-1\right| k^{-\frac{p(q-1)}{q+1-p}} \frac{r^{N}}{N}
\end{aligned}
$$

Hence, there exists a positive constant $c$ independent of $k$ such that

$$
\left|v_{k}^{\prime}(r)\right|^{p-1} \leqslant c r\left(1+k^{-\frac{p(q-1)}{q+1-p}}\right), \quad \text { for all } r \geqslant 0 .
$$

Using (4.12) and (4.15), we see that for $k \geqslant 1, v_{k}$ and $v_{k}^{\prime}$ are uniformly bounded on any compact subsets $K$ of $[0,+\infty)$. So, by the Arzela-Ascoli 
theorem and a standard diagonal argument, there is a sequence $k_{n} \longrightarrow+\infty$ and a $\mathcal{C}^{1}$ continuous function $v:[0,+\infty) \longrightarrow \mathbb{R}$ such that $v_{k_{n}} \underset{k_{n}}{\longrightarrow} \vec{\longrightarrow} v$ uniformly in $C^{1, \alpha}(K)$. It follows from (4.13) that $v$ satisfies

$$
\left\{\begin{array}{l}
r^{N-1}\left|v^{\prime}\right|^{p-2} v^{\prime}(r)=-\int_{0}^{r} s^{N-1}|v|^{q-1} v(s) d s, \text { for any } r>0 \\
v(0)=1 \text { and } v^{\prime}(0)=0 .
\end{array}\right.
$$

Consequently, we have

$$
r^{1-N}\left(r^{N-1}\left|v^{\prime}\right|^{p-2} v^{\prime}(r)\right)^{\prime}+|v|^{q-1} v(r)=0, \quad \text { for } r>0 .
$$

Now it remains to show that $v$ is strictly positive. Indeed, since $v_{k} \geqslant 0$ then $v \geqslant 0$. On the other hand (4.16) implies that $v$ is strictly decreasing. Thus, we have $v>0$.

Proposition 4.3.- Assume $q>\frac{p}{N}+p-1$. If for some $\gamma>0$ the solution $u$ of $(P)$ is non positive then, either $u$ has a compact support or there is a positive constant $M$ such that $u$ has at most one zero in $[M,+\infty)$.

Proof. - Since $\lim _{r \rightarrow+\infty} u(r)=0$, there exists $M>0$ such that

$$
|u(r)|^{q-1}<\frac{N(q+1-p)}{p(q-1)}-\frac{1}{q-1}, \text { for } r \geqslant M .
$$

Suppose that there exist two consecutive zeroes such that $M \leqslant x<$ $y$. Integrating equation (1.1) from $x$ to $y$ therefore gives

$$
\begin{gathered}
y^{N-1}\left|u^{\prime}\right|^{p-2} u^{\prime}(y)-x^{N-1}\left|u^{\prime}\right|^{p-2} u^{\prime}(x)= \\
\int_{x}^{y}\left[\frac{N(q+1-p)}{p(q-1)}-\frac{1}{q-1}-|u|^{q-1}(s)\right] s^{N-1} u(s) d s .
\end{gathered}
$$

Suppose that $u(r) \geqslant 0$ for $x<r<y$ (The same argument can be applied when $u(r) \leqslant 0)$ and distinguish two cases.

(i) $u^{\prime}(x) u^{\prime}(y)=0$. Then from the decreasing of the function energy $E$ given by (1.47), $u$ must have a compact support.

(ii) $u^{\prime}(x) u^{\prime}(y) \neq 0$. Then, $u^{\prime}(x)>0$ and $u^{\prime}(y)<0$. Now using (4.18), the right side of (4.19) is strictly positive while the left side is strictly negative, which yields a contradiction. Thus, two such zeroes can not exist. 
On the radial solutions of a degenerate quasilinear elliptic equation in $\mathbb{R}^{N}$

As a consequence of the previous propositions, we obtain the following result.

Corollary 4.1. - Assume $q>\frac{p}{N}+p-1$. Then any non positive solution of $(P)$ has either a compact support or a finite number of zeroes.

Proof - Let $u$ be a non positive solution of $(P)$. Then, from proposition 4.3, there exists a positive constant $R$ such that all zeroes of $u$ are in $[0, R]$. suppose that there is an increasing sequence $\left(x_{n}\right)$ of zeroes of $u$ and let $x=\lim _{n \rightarrow+\infty} x_{n}$. Then, for any $n \in \mathbb{N}$, there exists $z_{n} \in\left(x_{n}, x_{n+1}\right)$ such that $u^{\prime}\left(z_{n}\right)=0$. This implies that $\lim _{n \rightarrow+\infty} z_{n}=x$. And so, $u(x)=$ $u^{\prime}(x)=0$. Hence $u$ has a compact support. This finishes the proof.

Acknowledgments. The authors would to express their thanks to professor J.I.DIAZ for its remarks as well as for his article [1]. They also would to thank the referee for helpful comments. This work was supported finantially by Centre National de Coordination et de Planification de la Recherche Scientifique et Technique PARS MI 29.

\section{Bibliographie}

[1] DIAZ J.I. and SAA J.E. - Uniqueness of very singular self-similar solution of a quasilinear degenerate parabolic equation with absorption, Publicacions Matemàtiques, Vol. 36 (1992), 19-38.

[2] GmirA A. - On quasilinear parabolic equation involving measure data, Asymptotic Analysis 3 (1990), 43-56.

[3] GUEDDA M. and Veron L. - Local and global properties of solutions of quasilinear elliptic equation, J. Diff. Eq. $76, \mathrm{n}^{\circ} 1$ (1988), 159-189.

[4] HarauX A. and Weissler F.B. - Non uniqueness for a semilinear initial value problem, Indiana Univ. Math. J., 31 (1982), 167-189.

[5] KAMIN S. and VAZQUEZ J.L. - Singular solutions of some nonlinear parabolic equations, J. d'Analyse Mathematique, Vol. 59 (1992), 51-74.

[6] NAITO Y. and YASHIDA K. - Damped oxillation of solutions for some nonlinear second order ordinary differential equation, Adv. in Math. Sciences and App., Gakkotosho, Tokyo, vol. $5, \mathrm{n}^{\circ} 1$ (1995), 239-248.

[7] Peletier L.A., Terman D. and Weissler F.B. - On the equation $\Delta u+\frac{1}{2} x \nabla u+$ $f(u)=0$, Arch. Rational Mech. Anal., 94 (1986), 83-99.

[8] Peletier L.A. and WANG J. - A very singular solution of a quasilinear degenerate diffusion equation with absorption, Trans. Am. Math. Soc., 307 (1988), 813-826. 
Betteoui Benyounes, Abdelilah Gmira

[9] PUCCI P., SERrIN J. - Continuation and limit properties for solutions of strongly non linear second order differential equations, Asymptotic Analysis, 4 (1991), 97-160. North-Holland.

[10] WeISSLER F.B. - Asymptotic Analysis of an ordinary differential equation and nonuniqueness for a semilinear partial differential equation, Archive for Rational Mech and Anal. 91 (1986), 231-245.

[11] YoshidA K. - "Functional Analysis", Grundlehren der mathematischen Wissens chaften 123. Springer-Verlag, New York. Sixth edition (1980). 